\title{
Beneficial and deleterious bacterial-host interactions in chronic wound pathophysiology
}

\author{
This article was published in the following Dove Press journal: \\ Chronic Wound Care Management and Research \\ 2 April 2015 \\ Number of times this article has been viewed
}

\author{
Chase Watters' \\ Tony T Yuan ${ }^{1,2}$ \\ Kendra P Rumbaugh ${ }^{3}$ \\ 'Oak Ridge Institute for Science \\ and Education, Oak Ridge, TN, \\ USA; ${ }^{2}$ Department of Biomedical \\ Engineering, University of Texas at \\ San Antonio, San Antonio, TX, USA; \\ ${ }^{3}$ Department of Surgery, Texas Tech \\ University Health Sciences Center, \\ Lubbock, TX, USA
}

\begin{abstract}
Chronic wounds represent a major health and financial burden. Although incredible advancements in wound management have been made in the last decade, the incidences of chronic wounds continue to increase due to a rise in biofilm-associated infections. The presence of biofilm causes chronic inflammation, leading to impaired healing rates and host mortality. This review describes the deleterious bacterial-host interactions, as well as the beneficial role of $\mathrm{pH}$ and probiotics in chronic wound infections.
\end{abstract}

Keywords: chronic wounds, biofilms, wound pH, Pseudomonas aeruginosa, Staphylococcus aureus, probiotics

\section{Definition of the wound biofilm; bacterial species that colonize chronic wounds}

The skin is the first line of defense for the body, providing protection against toxins, microorganisms, and chemicals in the environment. Thus, the loss of skin integrity can result in substantial physiologic imbalance and significant disability or death. The prevalence of chronic wounds associated with the loss of skin is an important concern within the health care field. ${ }^{1}$ It is estimated that approximately seven million patients have some form of chronic wounds/ulcers that are associated with diabetes or pressure..$^{2-4}$ The health care cost for chronic wounds is estimated to be as high as $\$ 20$ billion annually in the United States. ${ }^{5-8}$ The current standard of care for chronic wounds consists of debridement, irrigation, moisture retentive bandages, and antimicrobial therapy. These treatment paradigms are aimed at promoting wound healing and the restoration of homeostasis.

Despite incredible advancements in the field of wound healing over the last decade, the prevalence and incidence of chronic wounds continues to rise ${ }^{6,7}$ Clinically, chronic wounds/ulcers are defined as any wound that is nonhealing after 30 days, and can be classified into three types of ulcers: vascular (eg, venous or arterial); diabetic; and pressure. ${ }^{9,10}$ These wounds are resistant to natural healing, and may require long-term medical care. ${ }^{10,11}$ Chronic wounds display delayed healing for a variety of reasons including diminished blood supply, uncontrolled inflammatory response, reduced re-epithelialization, and the presence of biofilm-associated infections. ${ }^{10,12}$

Biofilms are characterized as aggregated communities of microbes attached to a surface and/or each other, embedded in an extracellular polymeric substance (EPS) matrix composed of microbial- and host-derived extracellular DNA, proteins, and polysaccharides. These communities are often polymicrobial and dynamic, consisting of diverse species that are continuously changing. ${ }^{13}$ Biofilms can be found in a
Correspondence: Kendra P Rumbaugh Department of Surgery, Texas Tech University Health Sciences Center, MS 83I2, 360I 4th Street, Lubbock, TX 79430, USA

Tel +l 8067433684

Fax +l 8067432113

Email kendra.rumbaugh@ttuhsc.edu 
number of places on and in the body including the teeth, gastrointestinal mucosa, nasal epithelium, and any implanted artificial surface (eg, orthopedic implants, artificial valves, and catheters). Though the impact of biofilms in the pathogenesis of wound infections remains debatable, certain bacterial species have clearly been shown to hinder wound healing. It is thought that wounds are first contaminated and then colonized by adherent replicating microorganisms, which do not cause tissue damage. When the bacterial load in the wound exceeds $10^{5}$ colony-forming units/gram tissue, ${ }^{14}$ the initial colonization is thought to have progressed to an infection. While microbial colonization of a wound alone does not prolong healing, ${ }^{15}$ the subsequent infection can lead to tissue damage. The classical symptoms of a chronic wound infection are pain, heat, edema, and purulence. However, more contemporary signs and symptoms include pain, foul odor, and wound breakdown. ${ }^{16}$

The microbial flora of chronic wounds encompasses a variety of microorganisms including aerobic and anaerobic Gram-negatives and Gram-positives, as well as fungi. ${ }^{17}$ Some of the most common causes of wound infections are the ESKAPE pathogens (Enterococcus faecium, Staphylococcus aureus, Klebsiella pneumoniae, Acinetobacter baumannii, Pseudomonas aeruginosa, and Enterobacter spp.). ${ }^{18}$ However, just as every tumor is different, every biofilm infection is a unique consortium of bacteria, fungi, and host components, which can vary greatly from the initial injury to a long-term nonhealing wound. Prior to injury, normal flora inhabit the skin and differ depending on the location - eg, sebaceous areas harbor Propionibacterium spp., while moist areas predominantly support Staphylococcus and Corynebacterium spp. and, surprisingly, dry areas foster many Gram-negative bacteria previously thought to rarely colonize the skin. ${ }^{19}$

Normal flora can easily colonize wounds and lead to wound infections, as reported by a recent clinical study examining the evolution of the microflora in burn wound infections. ${ }^{20}$ In this study, $33 \%$ of patients' wounds were already colonized upon admission. About $50 \%$ of these burn wounds were found to be colonized by $S$. aureus, while the other $50 \%$ harbored Gram-negative organisms such as $P$. aeruginosa, as well as Acinetobacter, Klebsiella, and Enterobacter spp., and Escherichia coli. ${ }^{20}$ However, as this study was conducted using standard culturing techniques (serial dilutions, and culturing on nutrient-rich agar plates), nonculturable microorganisms were not considered. In contrast, utilizing a variety of molecular techniques (pyrosequencing, denaturing gradient gel electrophoresis, and full ribosome shotgun sequencing), the Wolcott laboratory reported the microbial populations from 30 patients with different types of chronic wound infections. ${ }^{21}$ Regardless of the type of wound (diabetic, pressure, or vascular), the most common genera found were Staphylococcus, Pseudomonas, Peptoniphilus, Enterobacter, Stenotrophomonas, Finegoldia, and Serratia spp. ${ }^{21}$ Strict and facultative anaerobic bacteria were shown to make up the majority of the microbial population (around 80\%) in the wounds, but ratios of strict/facultative anaerobes differed based on the wound type: diabetic $(25 \% / 65 \%)$; pressure (58\%/25\%); and venous (5\%/75\%).

With high-throughput sequencing techniques becoming cheaper and more accessible to health care providers, a whole host of new microbes are now becoming associated with disease. Undoubtedly, large amounts of new information will be generated concerning which microbes are harmful, beneficial, and/or neutral in the chronic wound. Meanwhile, the purpose of this review is to examine recent evidence concerning the deleterious interactions between bacteria and wound tissue, discuss the possibility of using beneficial bacteria to treat wound infections, and consider the translational potential of current studies to optimize future wound management approaches.

\section{Deleterious bacterial-host interactions in chronic wound pathophysiology}

Bacterial biofilms are thought to delay wound healing for a variety of reasons, namely by shifting the wound immune response toward chronic inflammation (Figure 1). In theory, as long as the biofilm is present, the immune system will try to remove it, resulting in prolonged inflammation with collateral damage to the host tissue. ${ }^{22}$ The host immune response is a complex multifaceted system that has been divided into the adaptive and innate immune systems. The innate immune response is made up of the skin barrier, commensal bacteria, the complement system, and both phagocytic (eg, macrophages and neutrophils) and nonphagocytic (eg, natural killer cells) leukocytes. The innate immune system recognizes a broad group of molecules specific to microbes such as lipopolysaccharide, peptidoglycan, and lipoteichoic acid via pattern recognition receptors, and is critical in fighting off bacterial infections and mounting the initial immune response to invading microbes. ${ }^{23}$ Once the epithelial skin barrier is compromised, the microbial skin flora are typically the first to contaminate, colonize, and potentially infect the wound. Tissue macrophages and other cells respond to these invading bacteria by releasing cytokines and chemokines, which stimulate polymorphonuclear neutrophil (PMN) 


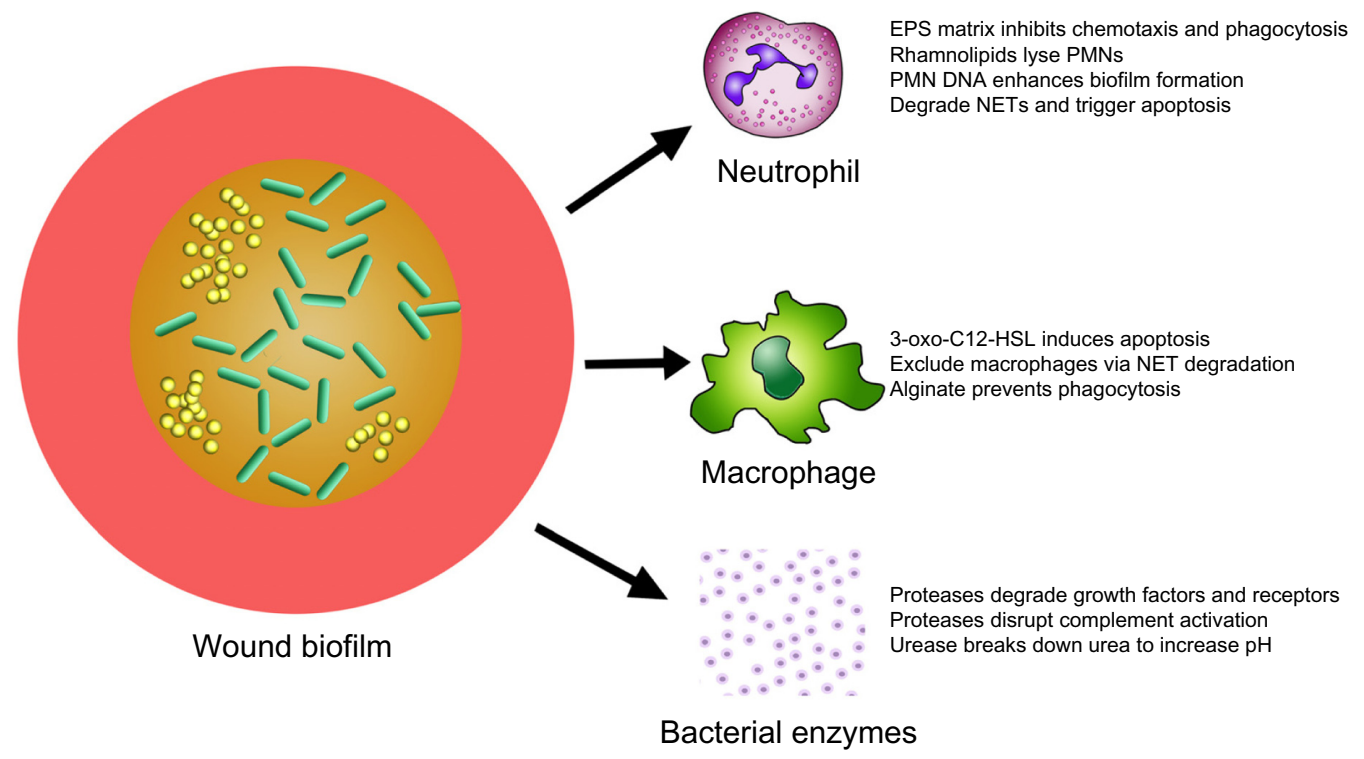

Figure I Deleterious actions of biofilms in chronic wounds.

Notes: Bacterial biofilms are thought to delay wound healing for a variety of reasons, including reducing local oxygen levels, mechanically inhibiting granulation, increasing tolerance to antimicrobials, and shifting the immune response toward chronic inflammation. Interactions between bacterial biofilms and dying or ineffective neutrophils and macrophages appear to significantly contribute to the chronic inflammatory state seen in chronic wound infections.

Abbreviations: EPS, extracellular polymeric substance; PMN, polymorphonuclear neutrophil; NET, neutrophil extracellular trap; 3-oxo-CI2-HSL, N-3-oxo-doedecanoylL-homoserine lactone.

chemotaxis to the wound site. One to 2 days later, macrophages migrate to the wound site to phagocytose bacterial cells, and for efferocytosis, which refers to the engulfment of apoptotic cells. Dendritic cells and macrophages then present antigens to the correct T-cells, which begins the cascade for subsequent antibody production. ${ }^{24}$ However, pathogenic bacteria have evolved numerous methods to evade the immune response in wound infections, which the following section will expound upon.

\section{Polymorphonuclear neutrophils and biofilms}

PMNs are critical to the host defense against bacteria, illustrated by the life-threatening bacterial sepsis problems associated with individuals who lack PMNs (neutropenia). ${ }^{25}$ Activated PMNs rapidly migrate to the site of injury, and persist there for 2-3 days. ${ }^{26} \mathrm{PMNs}$ do not recognize specific antigens, but instead recognize evolutionary conserved molecules shared by numerous bacterial species such as lipopolysaccharides and bacterial DNA. ${ }^{27}$ PMNs are able to recognize bacterial DNA and complex carbohydrates, both of which are essential components of the biofilm EPS matrix. ${ }^{28,29}$ PMNs have been shown to kill bacteria by four specific mechanisms: phagocytosis; the release of microbicidal compounds through degranulation; reactive oxygen species (ROS) generation; and the formation of neutrophil extracellular traps (NETs). ${ }^{30}$
While most of the previous work examining the bactericidal abilities of neutrophils was performed with planktonic bacteria, there has been a surge in recent published studies examining the relationship of PMNs and biofilms. The majority of work on PMNs and biofilm interactions has been done with $P$. aeruginosa and $S$. aureus biofilms. ${ }^{31}$ When $P$. aeruginosa biofilms were incubated with PMNs in vitro, the PMNs settled onto the biofilms, but they exhibited very little bactericidal activity or movement. ${ }^{32}$ One explanation for this observation was that the alginate present in $P$. aeruginosa's EPS matrix inhibited PMN phagocytosis and chemotaxis. ${ }^{33}$ Rhamnolipids are another mechanism by which $P$. aeruginosa biofilms antagonize PMNs and are actively produced in the biofilm mode of growth. ${ }^{34,35}$ Jensen et $\mathrm{al}^{36}$ observed that rhamnolipids, produced by biofilms, rapidly lysed human PMNs in vitro. This group went on to show that $P$. aeruginosa upregulated rhamnolipid production in response to the presence of PMNs, signifying that $P$. aeruginosa actively recognizes and responds to these immune cells. $P$. aeruginosa biofilms have also been shown to produce rhamnolipids in vivo, resulting in increased PMN lysis. ${ }^{37}$

There is also considerable in vitro and in vivo evidence that lysed PMNs enhance $P$. aeruginosa biofilm formation. For example, PMNs were shown to eradicate planktonic $P$. aeruginosa cells, while their presence increased the number of biofilm cells. ${ }^{37}$ The authors concluded that $P$. aeruginosa utilized the actin and DNA from lysed PMNs 
to fortify their biofilms. In addition, neutrophil-enhanced $P$. aeruginosa biofilms were more tolerant to antipseudomonal antibiotics. When these biofilms were treated with DNase, the biofilm was disrupted and there was an increase in planktonic cells. ${ }^{38}$ Furthermore, $P$. aeruginosa's ability to take advantage of neutrophils was recently demonstrated in a murine type- 1 diabetic wound infection model. ${ }^{39}$ Diabetic mice on insulin therapy that were wounded and infected with $P$. aeruginosa were shown to have increased PMN migration as compared to both diabetic and nondiabetic mice. However, this increased migration did not lead to increased bacterial clearance. Rather, increased neutrophil migration in this group of mice resulted in $P$. aeruginosa biofilms that contained more DNA and exhibited increased tolerance to the antibiotic gentamicin. ${ }^{39}$

Further evidence of the deleterious interactions between biofilms and PMNs came from Nguyen et al, ${ }^{40}$ who utilized a type 2 diabetic murine wound infection model to show that the presence of $S$. aureus biofilms significantly reduced neutrophil oxidative activity, leading to higher bacterial load and decreased healing rates. While $S$. aureus induces NETosis in PMNs, it was recently shown that $S$. aureus is able to degrade NETs to deoxyadenosine, triggering the caspase-3mediated cell death of immune cells. ${ }^{41}$ In addition, clinical staphylococcal infections seem to be correlated to reduced neutrophil apoptosis in diabetic patients, which leads to prolonged tumor necrosis factor- $\alpha$ production and reduced neutrophil clearance. ${ }^{42}$ In summary, PMNs are effective at killing planktonic cells, ${ }^{43}$ but biofilms appear to resist the bactericidal effects of PMNs, and may even benefit from the cellular debris left in their wake. It is clear from these recent studies that interactions between bacterial biofilms and dying or ineffective neutrophils contribute strongly to the chronic inflammatory state seen in chronic wound infections.

\section{Macrophages and biofilms}

Following the migration of PMNs to the site of injury, another innate immune cell population arrives: the macrophages. Macrophages arrive 2-3 days following tissue damage to challenge any microbial invaders, which the PMNs have not eradicated. Macrophages also phagocytize apoptotic/necrotic cells in a process called efferocytosis. ${ }^{44}$ Macrophages kill bacterial cells by phagocytosis followed by the production of ROS, but the interactions of macrophages with biofilms are less well characterized than those of PMNs. In 2003, it was observed that $\mathrm{N}$-(3-oxododecanoyl)-L-homoserine lactone (3-oxo-C12-HSL), a quorum-sensing molecule produced by $P$. aeruginosa, induced apoptosis in macrophages. ${ }^{45}$
However, 3-oxo-C12-HSL has also been shown to activate phagocytosis, while not affecting ROS production. ${ }^{46}$ When biofilms were incubated with macrophages in vitro, those lacking alginate were eradicated by macrophages in 4 hours. The killing of these biofilm cells was mediated through phagocytosis, and was dependent on the presence of interferon- $\gamma \cdot{ }^{47} S$. aureus has been shown to reduce macrophage numbers by releasing nuclease and adenosine, which degrade NETs and exclude macrophages from abscesses. ${ }^{41}$ Based upon the literature, $P$. aeruginosa biofilms seem less protected from macrophages, though there are various mechanisms that $P$. aeruginosa and $S$. aureus employ to diminish their effectiveness.

\section{Bacterial proteases and wound tissue}

Pathogenic bacteria produce a wide range of serine, cysteine, and metalloproteases that have intracellular and/or extracellular targets and can aid in colonization, the evasion of host defense, dissemination of bacteria, and tissue degradation. ${ }^{48,49}$ Intracellular proteases are typically important in cellular and metabolic processes, while extracellular proteases are important in the ability of bacteria to interact with the host environment, such as absorbing and utilizing hydrolytic products. ${ }^{50}$ In addition, bacterial proteases have the capability to degrade host growth factors and their receptors. ${ }^{51}$ For example, Laarman et $\mathrm{l}^{52}$ showed that $P$. aeruginosa alkaline protease was capable of disrupting complement activation by inhibiting opsonization of the bacteria by $\mathrm{C} 3 \mathrm{~b}$ and the subsequent formation of $\mathrm{C} 5 \mathrm{a}$. This protease has also been shown to help $P$. aeruginosa evade phagocytosis in the lung by degrading surfactant protein-A. ${ }^{53}$

\section{Wound $\mathrm{pH}$ and biofilm formation}

The acidic pH of the skin ( $\mathrm{pH} 4-6)$, known as the acid mantle, is one of the key mechanisms that protects the skin from microbial infections. In chronic wounds, the destruction of underlying tissue releases bodily fluids ( $\mathrm{pH} 7.4$ ) that shift the $\mathrm{pH}$ of the wound toward neutral/alkaline conditions. ${ }^{54}$ The $\mathrm{pH}$ of the wound environment has been shown to be an accurate method of predicting nonhealing wounds (Figure 2). Clinical studies have suggested that if the wound environment does not shift toward an acidic $\mathrm{pH}$ early in treatment, the probability of the wound failing to heal and requiring a skin graft increases significantly. For example, a randomized clinical study found that out of 25 venous ulcers, those that were considered "non healing" had a $\mathrm{pH}$ of 7.42, while wounds that had a $\mathrm{pH}$ of 6.91 healed. ${ }^{55}$ The relationship of wound healing and $\mathrm{pH}$ was further explored in a clinical study that examined the $\mathrm{pH}$ of 50 chronic and acute wounds. Overall, the authors reported 


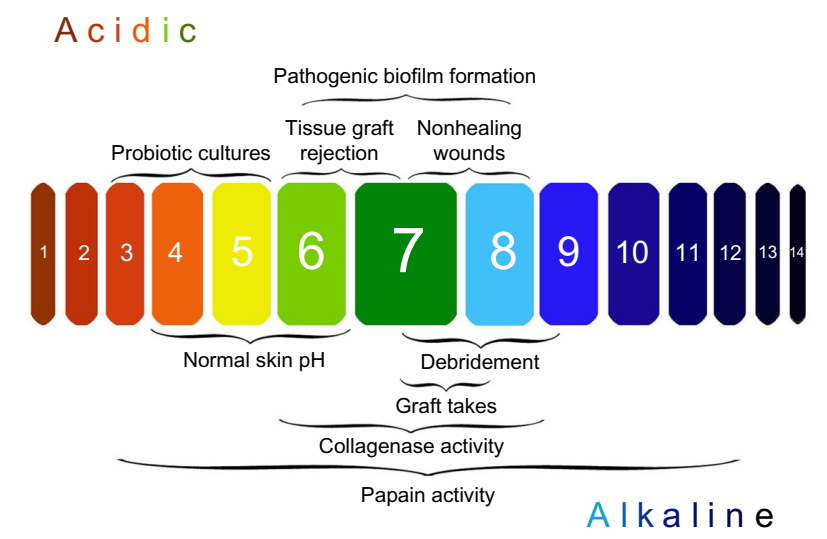

Figure 2 The importance of managing $\mathrm{pH}$ in chronic wound infections.

Notes: The acidic $\mathrm{pH}$ of the skin is one of the key mechanisms that protect it from microbial infections. In wounds, increasingly alkaline $\mathrm{pH}$ is a predictor that they will become nonhealing or chronic. However, wounds that are too acidic are less likely to successfully accept grafts. The activities of most biological and enzymatic therapies have $\mathrm{pH}$ optimums, and numerous factors can shift wound $\mathrm{pH}$ including debridement and bacterial colonization.

that healing wounds shifted below a $\mathrm{pH}$ of 8.0, while chronic wounds remained above a $\mathrm{pH}$ of 8.5. ${ }^{56}$

There are numerous factors that shift the wound toward an alkaline $\mathrm{pH}$, including lowered oxygen tension, stage of healing, debridement, maggot therapy, and bacterial colonization (Figure 2). ${ }^{54}$ For example, Proteus mirabilis, Klebsiella spp., and P. aeruginosa produce urease, an enzyme that catalyzes the formation of ammonia from urea. The release of alkaline ammonia promotes bacterial attachment and growth, as many wound pathogens require neutral to alkaline $\mathrm{pH}$ environments to form biofilms. ${ }^{57}$ Additionally, an alkaline $\mathrm{pH}$ reduces the release of oxygen supporting the growth of anaerobic bacteria. For more information on the impact of $\mathrm{pH}$ on wound healing and biofilm formation, refer to this recent and thorough review. ${ }^{57}$ Increased wound $\mathrm{pH}$ has major implications for delayed wound healing, and the next section will focus on bacterial therapies that target this problem in chronic wound infections.

\section{Beneficial bacterial-host interactions that promote chronic wound healing}

Physicians face many challenges in the management of nonhealing chronic wounds including disrupting bacterial biofilms, the global rise in antibiotic resistance, excessive inflammation, and an alkaline wound environment. Thus, the ideal therapy for wound infections is one that can reduce wound $\mathrm{pH}$, dampen the immune response and target the bacterial infection. One promising and relatively unexplored treatment that meets these criteria is the use of probiotic therapy. Utilizing commensal probiotics has shown promise in preventing and treating gut, oral, and urinary tract bacterial infections. ${ }^{58}$ As probiotics are typically bacteria that reside in the gut, they prefer an acidic environment and grow best in $\mathrm{pH} 3-5$. It has been proposed that, because probiotics can compete with pathogens for host tissue binding sites, stimulate the release of anti-inflammatory cytokines, lower $\mathrm{pH}$, release antimicrobial compounds, and deactivate the virulence factors of bacterial pathogens, they are excellent candidates for promoting wound healing in diabetic foot ulcers. ${ }^{59}$ The following section will focus on probiotic studies conducted in vitro and in vivo.

\section{Battling wound pathogens with probiotics}

Among the ESKAPE pathogens, probiotic therapies have predominantly been utilized against $S$. aureus and $P$. aeruginosa . The primary probiotic bacterial species that have been tested therapeutically include numerous Lactobacillus spp., Bifidobacterium spp., and Propionibacterium spp. For example, Lactobacillus casei and Lactobacillus acidophilus inhibited the growth of ten clinical strains of $S$. aureus by up to $99 \%$ after a 24-hour coculture. ${ }^{60}$ In the same study, L. acidophilus also exhibited antimicrobial activity against 5/6 ESKAPE pathogens isolated from burn wounds. ${ }^{60}$ In another study, $S$. aureus biofilms were reduced by $11-17 \mathrm{~mm}$ using the spot plate assay by several Lactobacillus spp. and Bifidobacterium spp. ${ }^{61}$ The proposed mechanism for the inhibitory effect on $S$. aureus lies in the production of organic acids (and the subsequent lowering of $\mathrm{pH}$ ), as this antagonistic effect was abated when culture supernatants were neutralized. ${ }^{61}$ As recently as 2014, propionic acid, a byproduct of Propionibacterium acnes, was shown to kill planktonic $S$. aureus by lowering the $\mathrm{pH}$ of the bacterial cytoplasm. ${ }^{62}$ Additionally, propionic acid exerted broad-spectrum activity against $E$. coli and the fungal pathogen Candida albicans, inhibiting their growth at concentrations $>10 \mathrm{mM}$. Other studies examining the efficacy of probiotics to treat $S$. aureus are described in this recent review. ${ }^{63}$

In 2010, Ramos et $\mathrm{al}^{64}$ provided some of the strongest evidence for the utilization of probiotics in the destruction of $P$. aeruginosa biofilms in vitro. The authors reported that acidic filtrates prepared from Lactobacillus plantarum supernatants disrupted biofilms made by a laboratory strain of $P$. aeruginosa by $43 \%$ and a $P$. aeruginosa clinical strain isolated from a wound by $35 \% .{ }^{64}$ Ramos et al ${ }^{65}$ went on to use L. plantarum supernatants to both disperse and inhibit $P$. aeruginosa biofilms, and indirectly reduce the production of numerous virulence factors (elastase, 
pyocyanin, rhamnolipids) ${ }^{65}$ Overall, these studies provide the groundwork for understanding the antibiofilm properties of probiotics. However, there remain many questions as to the antimicrobial efficacy of probiotics against wound pathogens and multispecies biofilms.

Another mechanism by which probiotics have been shown to enhance wound healing is by protecting the host from pathogens like $S$. aureus and dampening the immune response. For example, the probiotic strains Lactobacillus reuteri and Lactobacillus rhamnosus were shown to protect human keratinocytes from $S$. aureus-induced cell death when applied prior to or concurrently with $S$. aureus. However, if $S$. aureus cells were incubated with keratinocytes for as little as 1 hour, the addition of Lactobacillus spp. did not provide any protection to the host skin cells. ${ }^{66}$ In another recent study, when L. rhamnosus was simultaneously incubated with $S$. aureus and PMNs (murine and human), there was a reduction in S. aureus-associated PMN death and NET formation. ${ }^{67}$ As ROS production is essential to NET formation, the authors next tested if pretreating PMNs with L. rhamnosus could prevent ROS production. L. rhamnosus incubation resulted in decreased ROS production when PMNs were activated with phorbol 12-myristate 13-acetate or $\mathrm{H}_{2} \mathrm{O}_{2}$. Some of the probiotic strains tested were able to activate NETosis alone, so this anti-inflammatory effect is clearly species specific. ${ }^{67}$ L. plantarum supernatants were similarly shown to protect human PMNs from $P$. aeruginosa-associated apoptosis/ necrosis via the destruction of $P$. aeruginosa quorum-sensing molecules. ${ }^{68}$ By disrupting pathogenic biofilms and protecting key cells in the wound environment, probiotics have clearly demonstrated efficacy to provide crucial support to wound healing in vitro.

\section{In vivo efficacy of probiotics to promote chronic wound healing}

While there are numerous studies investigating the impact of probiotics on wound pathogens in vitro, far fewer have examined their efficacy in vivo. Of the studies performed, by far the most common approach has been to apply a probiotic species topically to wounds, either prophylactically or concurrently with infection, and then monitor wound healing and/or bacterial load. For example, the ability of $L$. plantarum to prevent either $S$. aureus or $P$. aeruginosa infection was investigated by treating mouse wounds with L. plantarum (cells or supernatant) plus $S$. aureus, $P$. aeruginosa, or the pathogens alone. ${ }^{69}$ Based on histopathological analysis from tissue taken 2 days post-treatment, the authors concluded that
L. plantarum had prevented wound infection. ${ }^{69}$ Similarly, $P$. acnes topical therapy was tested in mouse incisions infected with $S$. aureus. ${ }^{70}$ Mice were given $5 \mathrm{~mm}$ wounds and treated with $P$. acnes that had been incubated for 3 days with either phosphate buffered saline (PBS) or glycerol. Wounds were then infected with $S$. aureus and followed for 3 days. Following the wound infection, the mice treated with $P$. acnes incubated in glycerol displayed a $60 \%$ decrease in $S$. aureus bacterial burden and increased wound healing as compared to treatment with $P$. acnes that had been incubated in PBS. ${ }^{70}$ This study suggests that glycerol fermentation and the production of compounds, like propionic acid, provide the mechanism for targeting wound pathogens. While the studies discussed earlier used probiotic therapy to treat or prevent wound infection, topical treatment with Lactobacillus brevis was also shown to increase wound healing and decrease inflammation over 21 days in uninfected rat wounds. ${ }^{71}$

Another probiotic application was based on the idea that Lactobacillus fermentum plus glucose produces nitrate and protons leading to nitric oxide gas (gNO) production, which can lower $\mathrm{pH}$, promote healing, and kill S. aureus. This concept was tested in a rabbit full thickness wound infection model. ${ }^{72}$ One day after the wounds were infected with $S$. aureus, they were treated for 21 days with an adherent patch containing lactic acid bacteria that produced gNO. The authors observed that wounds treated with the gNO patch were 2.52 times more likely to close and displayed modest histological improvements, as compared to wounds treated with control patches. While some of the work performed in animal models is encouraging, as of yet, there have been no studies treating pre-established in vivo biofilms with topical probiotic therapeutics. Clearly there is much work to be done in this field, examining different pathogens, various probiotic strains, and optimizing treatment strategies.

\section{Clinical studies examining the efficacy of probiotics to enhance wound healing}

While mechanistic studies examining the use of topical probiotics on wounds in animal models is lacking, there have been some very thorough and informative clinical studies. For example, a study conducted in Argentina evaluated the use of topical L. plantarum cultures to treat infected seconddegree and noninfected third-degree burns. ${ }^{73}$ The probiotic therapy was compared to silver sulfadiazine for the treatment of 80 total burn wounds $(38$ L. plantarum treatments versus 42 silver sulfadiazine treatments), and the markers of clinical effectiveness measured were bacterial load and 
wound healing rates. L. plantarum cultures were applied directly to the wounds for 10 days, and the patients receiving the probiotic topical therapy did not receive any other form of antimicrobial treatment. The other group of patients received a daily antiseptic bath with $0.5 \%$ chlorhexidine and treatment with silver sulfadiazine cream daily for 10 days. While there were no significant differences in bacterial load or healing rates observed between the groups, there were also no cases of sepsis in either treatment group, suggesting that L. plantarum can be safely used in humans and function similarly to the antimicrobial standard of care treatments for slow-healing burn wound infections. ${ }^{73}$ Another study was performed by the same research group examining the impact of topical L. plantarum cultures on 14 diabetic ulcers and 20 nondiabetic ulcers. ${ }^{74}$ Although no difference was observed between groups, treatment with topical L. plantarum reduced the numbers of bacteria, neutrophils, apoptotic/necrotic cells, and promoted wound healing.

The most recent clinical probiotic wound study was also conducted by Valdez et al and reported in a 2013 book chapter. ${ }^{75}$ In this study, type 2 diabetics with foot ulcers were treated with topical L. plantarum cultures (number $[\mathrm{n}]=20$ ) or with standard debridement $(n=10)$ over the course of 30 days, and then the patients were followed for 20 days posttreatment. The investigators reported that treating the diabetic foot ulcers with L. plantarum doubled the rates of wound healing, granulation, and bacterial load reduction. ${ }^{75}$ Overall, these clinical studies provide a great deal of optimism for the utilization of probiotic therapy in wound care management. While much optimization and evaluation remain to be done, the studies conducted thus far provide an excellent foundation for the clinical proof of principle for this therapy.

\section{Applying current knowledge to optimize the management of chronic wounds}

Throughout this review, we have discussed biofilms, the bacterial pathophysiology of wounds, probiotics, and wound healing, but how does this information translate to the clinic? One of the main translational concepts presented was the importance of $\mathrm{pH}$ in wound healing (Figure 2). The measurement of wound $\mathrm{pH}$ is an easy and important, but often overlooked, indicator of nonhealing wounds. Nonhealing wounds typically have a $\mathrm{pH}$ that is neutral to slightly alkaline, ${ }^{57}$ and from the experimental evidence, it appears that attempts should be made to reduce $\mathrm{pH}$ and shift the wound toward an acidic environment. This was found to be especially true when the $\mathrm{pH}$ of 30 burn wounds was measured following a second dressing change. ${ }^{76}$ Those wounds that went on to heal had a mean $\mathrm{pH}$ of 7.32 , while the mean $\mathrm{pH}$ was 7.73 in nonhealing wounds that required skin grafting.

This review also covered the use of probiotics to lower wound $\mathrm{pH}$, which theoretically will lead to decreased biofilm formation and persistence of pathogens, ${ }^{57}$ reduced apoptosis ${ }^{67,68}$ and migration of PMNs,${ }^{57}$ and will ultimately shift wounds toward the healing spectrum. However, there are some concerns with the use of probiotics to treat wounds, specifically concerning septicemia caused by the topical application of Lactobacillus spp. Although there were no reports of septicemia in the clinical wound studies that used L. plantarum topically, ${ }^{73-75}$ Lactobacillus septicemia is possible in severely immunocompromised individuals, and seems to be strain specific. Two retrospective studies comprising a total of 260 cases of Lactobacillus septicemia noted that the top causative strain was L. rhamnosus. ${ }^{77,78}$ Thus, there is a clear need for investigators to conduct future probiotic studies to carefully consider published data before choosing their probiotic strains and study parameters.

Although the use of probiotics may take some time to become widely accepted, there are therapeutics already available, which can lower $\mathrm{pH}$ and promote wound healing namely, honey and acidified nitrate creams. ${ }^{57}$ Recently, an acidic therapeutic 3\% citric acid solution was applied to diabetic and nondiabetic wounds and compared to the alkaline therapeutic, Eusol. In both the diabetic and nondiabetic patients, the $3 \%$ citric acid solution reduced the length of treatment time to nearly half that of the Eusol-treated groups. ${ }^{79}$

While debridement is essential in managing nonhealing wounds, it should also be noted that any form of debridement seems to immediately shift the wound toward an alkaline $\mathrm{pH}$ (Figure 2) due to the increased perfusion of blood components into the wound site. ${ }^{54}$ In addition, medicinal maggots used for debridement release ammonium bicarbonate as a byproduct, which potentially shifts the wound environment toward alkalinity. ${ }^{57}$ Though maggot debridement therapy is reported to be an effective wound therapeutic, there remains a clear gap on the impact of this therapy on wound $\mathrm{pH}$. Overall, there is a therapeutic window for the application of the aforementioned acidic agents following debridement to promote wound healing.

The efficacy of enzymatic debridement and antibiotics are also closely tied to wound $\mathrm{pH}$. For instance, enzymatic debridement agents like papain have a $\mathrm{pH}$ range of 3-12 (optimum $\mathrm{pH}$ : 7), while collagenase has an optimum $\mathrm{pH}$ range of $6-8$ 
(Figure 2), but fibrinolysis used in conjunction with DNase only has $\mathrm{pH}$ activity ranging from 4.5 to $5.5 .{ }^{54}$ Additionally, the impact of $\mathrm{pH}$ on new fluoroquinolones has recently been explored and it appears that acidic $\mathrm{pH}$ environments enhance the antimicrobial activity of delafloxacin ${ }^{80}$ and finafloxacin ${ }^{81}$ against $S$. aureus, but acidic environments decrease the antimicrobial activity of monofloxacin ${ }^{81}$ and ciprofloxacin. ${ }^{80}$ Thus, the optimal $\mathrm{pH}$ for different enzymatic and antibiotic agents needs to be carefully considered before applying these treatments to wounds.

Finally, wound $\mathrm{pH}$ also appears to be an important factor influencing grafting success. Several studies of burn and chronic wounds have noted that tissue rejection is more likely when wounds are acidic, ${ }^{54,76}$ and the more alkaline the wound, the better the likelihood that the skin graft will take (Figure 2). ${ }^{82}$ For example, in 18 different wounds requiring grafts, patients with a wound $\mathrm{pH}$ below 7.4 experienced $0 \%$ graft acceptance, while those above this cutoff experienced $99 \%$ graft acceptance. ${ }^{56}$ Thus, acidic $\mathrm{pH}$ in the wound is not always beneficial and must be adjusted and evaluated properly based on the required therapy.

\section{Conclusion}

Chronic wounds persist for a variety of reasons, but the role of bacterial biofilms in preventing healing seems very clear. The continuous presence of biofilms results in a chronic state of inflammation in the wound. Collateral tissue damage occurs as the immune system tries to remove the biofilm, and this often increases bacterial pathogenesis by stimulating growth, spread, or even invasion of the pathogen. Pathogens in a wound can contribute to increased wound $\mathrm{pH}$, cause neutrophil destruction, reduce macrophage infiltration, and decrease oxygen tension, all of which contribute to delayed wound healing. Manipulating the microbial ecology of wounds with probiotics appears to be a promising and cost-effective therapy with the potential to lower wound $\mathrm{pH}$, increase oxygen tension, reduce inflammation, and prevent or disrupt infection by pathogens.

\section{Acknowledgments}

Wound infection research in the Kendra P Rumbaugh laboratory is supported by grants from the National Institute of Allergy and Infectious Diseases (AI105763 and AI107570) and the US Army Research Office (grant 62507-LS). This review was supported in part by an appointment to the Postgraduate Research Participation Program administered by the Oak Ridge Institute for Science and Education. Special thanks to Katie Farris for her technical and artistic contributions to the review figures.

\section{Disclosure}

The authors report no conflicts of interest in this work.

\section{References}

1. Hay RJ, Johns NE, Williams HC, et al. The global burden of skin disease in 2010: an analysis of the prevalence and impact of skin conditions. J Invest Dermatol. 2014;134(6):1527-1534.

2. Sen CK, Gordillo GM, Roy S, et al. Human skin wounds: a major and snowballing threat to public health and the economy. Wound Repair Regen. 2009;17(6):763-771.

3. Kim PJ, Evans KK, Steinberg JS, Pollard ME, Attinger CE. Critical elements to building an effective wound care center. J Vasc Surg. 2013;57(6):1703-1709.

4. Sun BK, Siprashvili Z, Khavari PA. Advances in skin grafting and treatment of cutaneous wounds. Science. 2014;346(6212):941-945.

5. Supp DM, Boyce ST. Engineered skin substitutes: practices and potentials. Clin Dermatol. 2005;23(4):403-412.

6. Cowan LJ, Stechmiller JK, Phillips P, Yang Q, Schultz G. Chronic wounds, biofilms and use of medicinal larvae. Ulcers. 2013;2013: 487024.

7. Schultz GS, Sibbald RG, Falanga V, et al. Wound bed preparation: a systematic approach to wound management. Wound Repair Regen. 2003;11 Suppl 1:S1-S28.

8. Fogerty MD, Abumrad NN, Nanney L, Arbogast PG, Poulose B, Barbul A. Risk factors for pressure ulcers in acute care hospitals. Wound Repair Regen. 2008;16(1):11-18.

9. Greenhalgh DG. Skin, soft tissue, and wound healing in the elderly. In: Yelon JA, Luchette FA, editors. Geriatric Trauma and Critical Care. New York, NY: Springer; 2014:37-44.

10. Demidova-Rice TN, Hamblin MR, Herman IM. Acute and impaired wound healing: pathophysiology and current methods for drug delivery, part 1: normal and chronic wounds: biology, causes, and approaches to care. Adv Skin Wound Care. 2012;25(7):304-314.

11. O'Donnell TF Jr, Passman MA, Marston WA, et al; Society for Vascular Surgery; American Venous Forum. Management of venous leg ulcers: clinical practice guidelines of the Society for Vascular Surgery ${ }^{\circledR}$ and the American Venous Forum. J Vasc Surg. 2014;60(2 Suppl):3S-59S.

12. Percival SL, Hill KE, Williams DW, Hooper SJ, Thomas DW, Costerton JW. A review of the scientific evidence for biofilms in wounds. Wound Repair Regen. 2012;20(5):647-657.

13. Vuotto C, Donelli G. Anaerobes in biofilm-based healthcare-associated infections. Adv Exp Med Biol. 2015;830:97-112.

14. Kirketerp-Møller K, Zulkowski K, James G. Chronic wound colonization, infection, and biofilms. In: Høiby N, Jensen PØ, Moser C, Høiby N, editors. Biofilm Infections. New York: Springer; 2011:11-24.

15. Edwards R, Harding KG. Bacteria and wound healing. Curr Opin Infect Dis. 2004;17(2):91-96.

16. Metcalf DG, Bowler PG. Clinician perceptions of wound biofilm. Int Wound J. Epub September 8, 2014.

17. Leake JL, Dowd SE, Wolcott RD, Zischkau AM. Identification of yeast in chronic wounds using new pathogen-detection technologies. JWound Care. 2009;18(3):103-104, 106, 108.

18. Thompson MG, Black CC, Pavlicek RL, et al. Validation of a novel murine wound model of Acinetobacter baumannii infection. Antimicrob Agents Chemother. 2014;58(3):1332-1342.

19. Kong HH, Segre JA. Skin microbiome: looking back to move forward. J Invest Dermatol. 2012;132(3 Pt 2):933-939.

20. Taneja N, Chari P, Singh M, Singh G, Biswal M, Sharma M. Evolution of bacterial flora in burn wounds: key role of environmental disinfection in control of infection. Int J Burns Trauma. 2013;3(2):102-107.

21. Dowd SE, Sun Y, Secor PR, et al. Survey of bacterial diversity in chronic wounds using pyrosequencing, DGGE, and full ribosome shotgun sequencing. BMC Microbiol. 2008;8:43.

22. Wolcott RD, Rhoads DD, Dowd SE. Biofilms and chronic wound inflammation. J Wound Care. 2008;17(8):333-341. 
23. Freudenberg MA, Tchaptchet S, Keck S, et al. Lipopolysaccharide sensing an important factor in the innate immune response to Gram-negative bacterial infections: benefits and hazards of LPS hypersensitivity. Immunobiology. 2008;213(3-4):193-203.

24. Davis P. The immunology of wound healing: the body as a battlefield. Wounds UK. 2008;4(4):54-69.

25. Fioredda F, Calvillo M, Bonanomi S, et al; Neutropenia Committee of the Marrow Failure Syndrome Group of the AIEOP (Associazione Italiana Emato-Oncologia Pediatrica). Congenital and acquired neutropenias consensus guidelines on therapy and follow-up in childhood from the Neutropenia Committee of the Marrow Failure Syndrome Group of the AIEOP (Associazione Italiana Emato-Oncologia Pediatrica). Am J Hematol. 2012;87(2):238-243.

26. Schultz GS, Ladwig G, Wysocki A. Extracellular matrix: review of its roles in acute and chronic wounds. World Wide Wounds. 2005.

27. Ozinsky A, Underhill DM, Fontenot JD, et al. The repertoire for pattern recognition of pathogens by the innate immune system is defined by cooperation between toll-like receptors. Proc Natl Acad Sci U S A 2000;97(25):13766-13771.

28. Underhill DM, Ozinsky A. Phagocytosis of microbes: complexity in action. Annu Rev Immunol. 2002;20:825-852.

29. El Kebir D, József L, Filep JG. Neutrophil recognition of bacterial DNA and Toll-like receptor 9-dependent and -independent regulation of neutrophil function. Arch Immunol Ther Exp (Warsz). 2008;56(1):41-53.

30. Kaplan MJ, Radic M. Neutrophil extracellular traps: double-edged swords of innate immunity. J Immunol. 2012;189(6):2689-2695.

31. Hänsch GM. Host defence against bacterial biofilms: "mission impossible"? ISRN Immunology. 2012;2012:853123.

32. Jensen PØ, Givskov M, Bjarnsholt T, Moser C. The immune system vs Pseudomonas aeruginosa biofilms. FEMS Immunol Med Microbiol. 2010;59(3):292-305.

33. Mulcahy LR, Isabella VM, Lewis K. Pseudomonas aeruginosa biofilms in disease. Microb Ecol. 2014;68(1):1-12.

34. Xavier JB, Kim W, Foster KR. A molecular mechanism that stabilizes cooperative secretions in Pseudomonas aeruginosa. Mol Microbiol. 2011;79(1):166-179.

35. Abdel-Mawgoud AM, Lépine F, Déziel E. Rhamnolipids: diversity of structures, microbial origins and roles. Appl Microbiol Biotechnol. 2010;86(5):1323-1336.

36. Jensen PØ, Bjarnsholt T, Phipps R, et al. Rapid necrotic killing of polymorphonuclear leukocytes is caused by quorum-sensing-controlled production of rhamnolipid by Pseudomonas aeruginosa. Microbiology. 2007;153(Pt 5):1329-1338.

37. van Gennip M, Christensen LD, Alhede M, et al. Interactions between polymorphonuclear leukocytes and Pseudomonas aeruginosa biofilms on silicone implants in vivo. Infect Immun. 2012;80(8):2601-2607.

38. Walker TS, Tomlin KL, Worthen GS, et al. Enhanced Pseudomonas aeruginosa biofilm development mediated by human neutrophils. Infect Immun. 2005;73(6):3693-3701.

39. Watters C, Everett JA, Haley C, Clinton A, Rumbaugh KP. Insulin treatment modulates the host immune system to enhance Pseudomonas aeruginosa wound biofilms. Infect Immun. 2014;82(1):92-100.

40. Nguyen KT, Seth AK, Hong SJ, et al. Deficient cytokine expression and neutrophil oxidative burst contribute to impaired cutaneous wound healing in diabetic, biofilm-containing chronic wounds. Wound Repair Regen. 2013;21(6):833-841.

41. Thammavongsa V, Missiakas DM, Schneewind O. Staphylococcus aureus degrades neutrophil extracellular traps to promote immune cell death. Science. 2013;342(6160):863-866.

42. Hanses F, Park S, Rich J, Lee JC. Reduced neutrophil apoptosis in diabetic mice during staphylococcal infection leads to prolonged Tnfo production and reduced neutrophil clearance. PLoS One. 2011;6(8): e23633.

43. Young RL, Malcolm KC, Kret JE, et al. Neutrophil extracellular trap (NET)-mediated killing of Pseudomonas aeruginosa: evidence of acquired resistance within the CF airway, independent of CFTR. PLoS One. 2011;6(9):e23637.
44. Khanna S, Biswas S, Shang Y, et al. Macrophage dysfunction impairs resolution of inflammation in the wounds of diabetic mice. PLoS One. 2010;5(3):e9539.

45. Tateda K, Ishii Y, Horikawa M, et al. The Pseudomonas aeruginosa autoinducer N-3-oxododecanoyl homoserine lactone accelerates apoptosis in macrophages and neutrophils. Infect Immun. 2003;71(10): 5785-5793.

46. Vikström E, Magnusson KE, Pivoriūnas A. The Pseudomonas aeruginosa quorum-sensing molecule $\mathrm{N}$-(3-oxododecanoyl)-L-homoserine lactone stimulates phagocytic activity in human macrophages through the p38 MAPK pathway. Microbes Infect. 2005;7(15):1512-1518.

47. Leid JG, Willson CJ, Shirtliff ME, Hassett DJ, Parsek MR, Jeffers AK. The exopolysaccharide alginate protects Pseudomonas aeruginosa biofilm bacteria from IFN-gamma-mediated macrophage killing. J Immunol. 2005;175(11):7512-7518.

48. McCarty SM, Cochrane CA, Clegg PD, Percival SL. The role of endogenous and exogenous enzymes in chronic wounds: a focus on the implications of aberrant levels of both host and bacterial proteases in wound healing. Wound Repair Regen. 2012;20(2):125-136.

49. Supuran CT, Scozzafava A, Mastrolorenzo A. Bacterial proteases: current therapeutic use and future prospects for the development of new antibiotics. Expert Opin Ther Pat. 2001;11(2):221-259.

50. Gupta R, Beg QK, Lorenz P. Bacterial alkaline proteases: molecular approaches and industrial applications. Appl Microbiol Biotechnol. 2002;59(1):15-32.

51. Schultz GS, Wysocki A. Interactions between extracellular matrix and growth factors in wound healing. Wound Repair Regen. 2009;17(2): 153-162.

52. Laarman AJ, Bardoel BW, Ruyken M, et al. Pseudomonas aeruginosa alkaline protease blocks complement activation via the classical and lectin pathways. J Immunol. 2012;188(1):386-393.

53. Kuang Z, Hao Y, Walling BE, Jeffries JL, Ohman DE, Lau GW. Pseudomonas aeruginosa elastase provides an escape from phagocytosis by degrading the pulmonary surfactant protein-A. PLoS One. 2011;6(11):e27091.

54. Schneider LA, Korber A, Grabbe S, Dissemond J. Influence of pH on wound-healing: a new perspective for wound-therapy? Arch Dermatol Res. 2007;298(9):413-420.

55. Roberts G, Chuley A, Mani R. The world milieu in venous ulcers further observations. Presented at the European Wound Management Association Conference. Stuttgart, Germany; 2005.

56. Shukla VK, Shukla D, Tiwary SK, Agrawal S, Rastogi A. Evaluation of $\mathrm{pH}$ measurement as a method of wound assessment. J Wound Care. 2007;16(7):291-294.

57. Percival SL, McCarty S, Hunt JA, Woods EJ. The effects of $\mathrm{pH}$ on wound healing, biofilms, and antimicrobial efficacy. Wound Repair Regen. 2014;22(2):174-186.

58. Hickson M. Examining the evidence for the use of probiotics in clinical practice. Nurs Stand. 2013;27(29):35-41.

59. Sonal Sekhar M, Unnikrishnan MK, Vijayanarayana K, Rodrigues GS, Mukhopadhyay C. Topical application/formulation of probiotics: will it be a novel treatment approach for diabetic foot ulcer? Med Hypotheses. 2014;82(1):86-88.

60. Karska-Wysocki B, Bazo M, Smoragiewicz W. Antibacterial activity of Lactobacillus acidophilus and Lactobacillus casei against methicillin-resistant Staphylococcus aureus (MRSA). Microbiol Res. 2010;165(8):674-686.

61. Tejero-Sariñena S, Barlow J, Costabile A, Gibson GR, Rowland I. In vitro evaluation of the antimicrobial activity of a range of probiotics against pathogens: evidence for the effects of organic acids. Anaerobe. 2012;18(5):530-538.

62. Wang Y, Dai A, Huang S, et al. Propionic acid and its esterified derivative suppress the growth of methicillin-resistant Staphylococcus aureus USA300. Benef Microbes. 2014;5(2):161-168.

63. Sikorska H, Smoragiewicz W. Role of probiotics in the prevention and treatment of meticillin-resistant Staphylococcus aureus infections. Int J Antimicrob Agents. 2013;42(6):475-481. 
64. Ramos AN, Peral MC, Valdez JC. Differences between Pseudomonas aeruginosa in a clinical sample and in a colony isolated from it: comparison of virulence capacity and susceptibility of biofilm to inhibitors. Comp Immunol Microbiol Infect Dis. 2010;33(3):267-275.

65. Ramos AN, Cabral ME, Noseda D, Bosch A, Yantorno OM, Valdez JC. Antipathogenic properties of Lactobacillus plantarum on Pseudomonas aeruginosa: the potential use of its supernatants in the treatment of infected chronic wounds. Wound Repair Regen. 2012;20(4):552-562.

66. Prince T, McBain AJ, O’Neill CA. Lactobacillus reuteri protects epidermal keratinocytes from Staphylococcus aureus-induced cell death by competitive exclusion. Appl Environ Microbiol. 2012;78(15): 5119-5126.

67. Vong L, Lorentz RJ, Assa A, Glogauer M, Sherman PM. Probiotic Lactobacillus rhamnosus inhibits the formation of neutrophil extracellular traps. J Immunol. 2014;192(4):1870-1877.

68. Ramos AN, Gobbato N, Rachid M, González L, Yantorno O, Valdez JC. Effect of Lactobacillus plantarum and Pseudomonas aeruginosa culture supernatants on polymorphonuclear damage and inflammatory response. Int Immunopharmacol. 2010;10(2):247-251.

69. Jabbar H, Al-Mathkhury F, Jabbar H, Al-Aubeidi AR. Probiotic effect of Lactobacilli on mice incisional wound infections. Journal of Al-Nahrain University. 2008;11(3):111-116.

70. Shu M, Wang Y, Yu J, et al. Fermentation of Propionibacterium acnes, a commensal bacterium in the human skin microbiome, as skin probiotics against methicillin-resistant Staphylococcus aureus. PLoS One. 2013;8(2):e55380.

71. Zahedi F, Heydari Nasrabadi M, Tajabadi Ebrahimi M, Aboutalebi H. Comparison of the effects of Lactobacillus brevis and Lactobacillus plantarum on cutaneous wound healing in rats. African Journal of Microbiology Research. 2011;5(24):4226-4233.

72. Jones M, Ganopolsky JG, Labbé A, et al. Novel nitric oxide producing probiotic wound healing patch: preparation and in vivo analysis in a New Zealand white rabbit model of ischaemic and infected wounds. Int Wound J. 2012;9(3):330-343.
73. Peral MC, Martinez MA, Valdez JC. Bacteriotherapy with Lactobacillus plantarum in burns. Int Wound J. 2009;6(1):73-81.

74. Peral MC, Rachid MM, Gobbato NM, Huaman Martinez MA, Valdez JC. Interleukin- 8 production by polymorphonuclear leukocytes from patients with chronic infected leg ulcers treated with Lactobacillus plantarum. Clin Microbiol Infect. 2010;16(3):281-286.

75. Valdez C, Ramos A, Fernadnez D, et al. Probiotics and their potential use in wound healing. In: Kitazawa H, Villena J, Alvarez S, editors. Probiotics: Immunobiotics and Immunogenics. Hoboken, NJ: Taylor \& Francis; 2013:298-335.

76. Sharpe JR, Booth S, Jubin K, Jordan NR, Lawrence-Watt DJ, Dheansa BS. Progression of wound $\mathrm{pH}$ during the course of healing in burns. J Burn Care Res. 2013;34(3):e201-e208.

77. Cannon JP, Lee TA, Bolanos JT, Danziger LH. Pathogenic relevance of Lactobacillus: a retrospective review of over 200 cases. Eur J Clin Microbiol Infect Dis. 2005;24(1):31-40.

78. Gouriet F, Million M, Henri M, Fournier PE, Raoult D. Lactobacillus rhamnosus bacteremia: an emerging clinical entity. Eur J Clin Microbiol Infect Dis. 2012;31(9):2469-2480.

79. Prabhu V, Prasadi S, Pawar V, Shivani A, Gore A. Does wound $\mathrm{pH}$ modulation with $3 \%$ citric acid solution dressing help in wound healing: a pilot study. Saudi Surgical Journal. 2014;2(2):38-46.

80. Lemaire S, Tulkens PM, Van Bambeke F. Contrasting effects of acidic $\mathrm{pH}$ on the extracellular and intracellular activities of the anti-gram-positive fluoroquinolones moxifloxacin and delafloxacin against Staphylococcus aureus. Antimicrob Agents Chemother. 2011;55(2):649-658.

81. Lemaire S, Van Bambeke F, Tulkens PM. Activity of finafloxacin, a novel fluoroquinolone with increased activity at acid $\mathrm{pH}$, towards extracellular and intracellular Staphylococcus aureus, Listeria monocytogenes and Legionella pneumophila. Int J Antimicrob Agents. 2011;38(1):52-59.

82. Sharpe JR, Harris KL, Jubin K, Bainbridge NJ, Jordan NR. The effect of pH in modulating skin cell behaviour. Br J Dermatol. 2009;161(3): 671-673.
Chronic Wound Care Management and Research

\section{Publish your work in this journal}

Chronic Wound Care Management and Research is an international, peer reviewed, open access, online journal publishing original research, reviews, editorials, and commentaries on the causes and management of chronic wounds and the major issues related to chronic wound management. Topics also include chronic wounds as comorbidities to other

\section{Dovepress}

conditions, patient adherence to therapy, and the economic burden of chronic wounds. The manuscript management system is completely online and includes a very quick and fair peer review system, which is all easy to use. Visit http://www.dovepress.com/testimonials.php to read real quotes from published authors. 